\title{
Evaluation of Dynamics of Pushing a Wheelchair Up or Down a Slope*
}

\author{
Kazuto MIYAWAKI**, Makoto SASAKI***, Takehiro IWAMI****, \\ Goro OBINATA $* * * * *$ and Yoichi SHIMADA**** \\ ${ }^{* *}$ Akita National College of Technology \\ 1-1 lijima, Bunkyou-cho, Akita-city, Akita, Japan 011-8511 \\ E-mail: miyawaki@akita-nct.jp \\ ***Iwate University, \\ 4-3-5 Ueda, Morioka-city, Iwate, Japan 020-8551 \\ E-mail: makotosa@iwate-u.ac.jp \\ ${ }^{\star * * *}$ Akita University, \\ 1-1 Gakuen-cho, Akita-city, Akita, Japan 010-8502 \\ E-mail: iwami@control.mech.akita-u.ac.jp, yshimada@hos.akita-u.ac.jp \\ ${ }^{* * * * *}$ Nagoya University, \\ Furo-cho, Chikusa-ku, Nagoya, Aichi, Japan 464-8603 \\ E-mail: obinata@mech.nagoya-u.ac.jp
}

\begin{abstract}
Japan's progressing aging society increasingly needs evaluation of equipment used for human assistance. Earlier studies have evaluated the use of wheelchairs. However, the manner in which the equipment moderates the generated consumption energy of helpers has not been described sufficiently. This study performs mechanical evaluation of a helper's walking using a wheelchair on a slope. We use the Musculoskeletal Model to estimate the joint moment and energy consumption. Results obtained with 14 volunteers who assisted these wheelchair experiments were considered for cases in which the energy consumption of the wheelchair increased by $13 \%$ from that of a normal gait when moving up an incline. This evaluation method is useful for developing practical assistance equipment.
\end{abstract}

Key words: Human Dynamics, Musculoskeletal Model, Joint Moment, Energy Consumption

\section{Introduction}

In progressing toward an elderly society, the increased use of welfare equipment by elderly people can enhance their quality of life every day. In existing circumstances, wheelchairs are the most widely used type of welfare equipment. Because residences and public facilities are incorporating barrier-free designs, the use of wheelchairs continues to increase steadily. Regarding evaluation of wheelchairs used in daily life ${ }^{(1)}$, a design guide incorporating operability is proposed for wheelchairs by which the capability at the time of their use is evaluated and developed quantitatively through trials to evaluate the upper-limb motion characteristics when operating a wheelchair ${ }^{(2)(3)}$. Using that guide, wheelchairs adapted to various cases have been developed, and environments have been prepared in which anyone can use a wheelchair that has been specifically tailored to their own specifications.

When using a wheelchair on a flat area, a wheelchair user can easily operate it. However, when using a wheelchair on a slope, it is desirable to use it with the support of a

*Received 22 Feb., 2011 (No. T2-08-0149) Japanese Original : Trans. Jpn. Soc. Mech. Eng., Vol.75, No.752, C (2009), pp.1033-1040 (Received 28 Feb., 2008) [DOI: 10.1299/jsdd.6.525]

Copyright (C) 2012 by JSME 
helper for safety and operability. Although the upper limit of the gradient of slopes is specified as $1 / 12$ by the Heart-Build Law, operation is necessary for assistance while pushing a wheelchair on a slope even within that specified range. In actual nursing care, alleviating the mental and physical burdens of helpers engenders improvement in the quality of assistance. Nevertheless, little quantitative investigation has been made of the physical burden that is put on helpers.

Conventional evaluation of welfare equipment was made primarily in two ways. One is sensory evaluation through a questionnaire survey or interview with users asked about their ease of use of the welfare equipment. Second is a strength test, endurance test, and safety test of the welfare equipment based on international standards. In this paper, however, we propose a quantitative evaluation of the physical burden on the lower limbs of a person from new viewpoints using welfare equipment.

Regarding assisting operations for pushing wheelchairs presented to date, Nishioka et al. performed subjective evaluation related to grip height as a basic investigation of operability and functionality of the helper and demonstrated that a grip height that allows the easiest operation is around $90 \%$ of the height of the point of elbow of each subject ${ }^{(4)}$. Similarly, Kawaguchi et al. performed sensory evaluation of the grip height of the assistance wheelchair and measured the operating posture and muscle potential of the upper limb using an operation measurement device and a muscle potential meter. Results show that pushing a wheelchair is easy if the grip is provided at $75 \%$ of the acromial height ${ }^{(5)}$. Koh et al. measured the heart rate of those who experienced assistance and those who did not, and evaluated psycho-physiological influences during assistance operations ${ }^{(6)}$. In Europe, Van Der Woude et al. evaluated the optimum grip height while forces for pushing an assistance wheelchair are measured by incorporating sensors to handle some of load of the assistance wheelchair ${ }^{(7)}$. Furthermore, Uchiyama et al. reported that characteristics when pushing force depending on a load and walking speed are regulated autonomously when using an electrically driven walking-action test device while a helper walks with pushing operations ${ }^{(8)}$. Consequently, sensory evaluation such as ease of pushing the assistant wheelchair and evaluation of burden exerted to the upper limb of the helper have been conducted. Nevertheless, no evaluation has been made to date for the lower limbs of a wheelchair helper during wheelchair assistance operations. In this study, we evaluated wheelchair pushing operations performed by a helper on up and down slopes where burdens borne by the lower limbs of helpers are regarded as substantial. Indices of the evaluation are the joint moment of the lower limb and muscle consumption energy assumed from muscular tension using Beale's optimization method, which is used frequently for gait analysis related to clinical rehabilitation.

\section{Human Analytic Model}

\subsection{Rigid Link Model}

A two-dimensional rigid rink model was used to represent walking helpers on a slope pushing a wheelchair up or down (Fig. 1). Respective links represent the foot, shank, thigh, body, arm, and wheelchair. This model was proposed by Yamazaki ${ }^{(9)}$ the measurement method was developed by Ehara et al. ${ }^{(10)(11)}$. A walking analysis using these links is now being considered for clinical application by Ohashi ${ }^{(12)}$.

The following were assumed for modeling the body:

(1) The motion of each body part in gait occurs on a vertical surface, including the direction of movement. The body rotation can be ignored.

(2) Each joint is assumed to be a rigid link and the center of gravity is positioned on an axis between joints.

(3) Each joint is considered a rotational joint of one axis. 
The vector of floor reaction force, gravity, and acceleration are used along with the ankle joint moment $M a$, knee joint moment $M k$, and hip joint moment $M h$ using the Newton-Euler equation of motion balancing force in horizontal and vertical components acting on each joint and moment, which results in Eqs. (1)-(3) ${ }^{(13)}$.

Link 1 (foot)

$$
\begin{aligned}
M_{a}= & -I_{1} \ddot{\theta}_{1}+f_{p} y_{1}+\left(f_{p}-m_{1} \ddot{x}_{1}\right)\left(y_{a}-y_{1}\right) \\
& +\left(n_{p} u-x_{1}\right)+\left(n_{p}-m_{1}\left(\ddot{y}_{1}+g\right)\right)\left(x_{1}-x_{a}\right)
\end{aligned}
$$

Link 2 (lower thigh)

$$
\begin{aligned}
M_{k}= & -I_{2} \ddot{\theta}_{2}+\left(f_{p}-m_{1} \ddot{x_{1}}\right)\left(y_{2}-y_{a}\right) \\
& +\left(f_{p}-m_{1} \ddot{x}_{1}-m_{2} \ddot{x}_{2}\right)\left(y_{k}-y_{2}\right) \\
& -\left(n_{p}-m_{1}\left(\ddot{y}_{1}+g\right)\right)\left(x_{2}-x_{a}\right) \\
& -\left(n_{p}-m_{1}\left(\ddot{y}_{1}+g\right)-m_{2}\left(\ddot{y}_{2}+g\right)\right)\left(x_{k}-x_{2}\right)-M_{a}
\end{aligned}
$$

Link 3 (thigh)

$$
\begin{aligned}
M_{h}= & -I_{3} \ddot{\theta}_{3}+\left(f_{p}-m_{1} \ddot{x}_{1}-m_{2} \ddot{x}_{2}\left(y_{3}-y_{k}\right)\right. \\
& +\left(f_{p}-m_{1} \ddot{x_{1}}-m_{2} \ddot{x}_{2}-m_{3} \ddot{x_{3}}\right)\left(y_{h}-y_{3}\right) \\
& -\left(n_{p}-m_{1}\left(\ddot{y}_{1}+g\right)-m_{2}\left(\ddot{y}_{2}+g\right)\right)\left(x_{k}-x_{3}\right) \\
& -\left(n_{p}-m_{1}\left(\ddot{y}_{1}+g\right)-m_{2}\left(\ddot{y}_{2}+g\right)\right. \\
& \left.-m_{3}\left(\ddot{y}_{3}+g\right)\right)\left(x_{3}-x_{k}\right)-M_{k} . . . .
\end{aligned}
$$

Therein, $f_{p}$ represents the floor reaction force in the horizontal component, $n_{p}$ signifies a floor reaction force in the vertical component, $x$ represents the coordinates of the center of link gravity in the horizontal component, $\ddot{x}$ represents the acceleration of the center of link gravity in the horizontal component, $y$ stands for the coordinates of the center of link gravity in the vertical component, $\ddot{y}$ denotes the acceleration of the center of link gravity in the vertical component, $m$ the link mass, $I$ denotes the inertia moment of the link, $\theta$ signifies the link angle, $\ddot{\theta}$ stands for the angular acceleration of the link, $g$ is the acceleration of gravity, and $u$ stands for coordinates in the horizontal component at the working point of floor reaction force. Subscript 1 denotes the foot, 2 is the lower thigh, 3 is the thigh, $a$ is the ankle, $k$ is the knee, and $h$ is the hip. The center of mass of the entire body link is called the center of body gravity. Ae et al. ${ }^{(14)}$ provide a useful description for the mass of the link and inertia moment.

\subsection{Musculoskeletal Model}

Yamazaki's musculoskeletal model ${ }^{(9)}$ (Fig. 2) incorporates the nine major muscles driving lower limb joints: (1) rectus femoris, (2) gluteus maximus, (3) hamstring muscles, (4) vatus, (5) biceps femoris, (6) gastrocnemius, (7) soleus, (8) tibialis anterior, and (9) musculus iliopsoas. Pedotti et al. ${ }^{(15)}$ proposed using the musculoskeletal model to find muscle tension. This method is consistent with electromyography results obtained by Ehara et al. ${ }^{(10)}{ }^{(11)}$. Pedotti's method was used to ascertain the muscle tension. Equations (4)-(6) show the relation between contraction generated by each muscle and the joint moment of each joint. 
$r 6 a \cdot F_{6}+r 7 \cdot F_{7}-r 8 \cdot F_{8}=M_{a}$

$-r 1 k \cdot F_{1}+r 3 k \cdot F_{3}-r 4 \cdot F_{4}$

$$
+r 5 \cdot F_{5}+r 6 \cdot F_{6 k}=M_{k}
$$

$$
\begin{aligned}
-r 1 h & \cdot F_{1}+r 2 \cdot F_{2} \\
& +r 3 h \cdot F_{3}+r 9 \cdot F_{9}=M_{h}
\end{aligned}
$$

In those equations, $F$ stands for individual muscle tension, $r$ signifies the lever arm length, and 1-9 denote muscle numbers. The lever arm runs from the center of joint revolution to a muscle point (Table 1). Using Eqs. (1)-(3) a value was obtained for each joint moment.

Six single joint muscles (the gluteus, vatus, biceps femoris soleus, tibialis anterior, and musculus iliopsoas) and three two-joint muscles spanning two muscles of the rectus femoris (the hamstring muscles and gastrocnemius) drive the ankle, knee, and hip. Three equations represent the balance between muscle tension and moment. They are fewer than the number of nine muscle tensions to be found. Muscle tension does not identify itself if nothing is done under these circumstances. Pedotti et al. showed that if evaluation function $\mathrm{Z}$ was set and minimized, actual muscle tension $F_{i}$ was obtained. Beale's method ${ }^{(16)}$, a form of nonlinear optimization, was used to find this solution. This practical method is distinctive in that memory usage is less, which makes it useful for high-speed optimization

$$
Z=\sum_{i=1}^{9}\left(\frac{F_{i}}{F_{\operatorname{maxi}}}\right)^{2} \quad 0 \leq F_{i} \leq F_{\max i} \ldots
$$

Therein, $F$ is the maximum muscle tension and $F_{\text {maxi }}$ is the muscle tension attributable to isometric contraction. A technique proposed by Ehara et al. ${ }^{(10)(11)}$, based on the relation between muscle tension and muscle contraction speed indicated by Hill ${ }^{(17)}$, was used to determine the energy a muscle consumes. The technique is consistent with values determined by the oxygen intake. The technique for determining the energy a muscle consumes, as proposed by Ehara ${ }^{(18)}$, is summarized below.

This technique uses the contraction speed to divide cases into (1) muscle contraction and (2) muscle isometric contraction or extension, and assumes the energy of a muscle as follows.

(1) When a muscle exhibits muscle tension attributable to isometric contraction $F_{0}$ because of neurostimulation, Hill's relation ${ }^{(16)}$ represents muscle tension $F$ that occurs when the muscle contracts in the same contraction at velocity $v(v>0)$ by Eq. (8).

$$
(F+A) \cdot(v+b)=\left(F_{0}+A\right) \cdot b
$$

In that equation, $A=a \cdot F_{0} / F_{\max }=0.25 F_{0}, b / L_{0}=0.9 / \mathrm{s}$. In addition, $a$ and $b$ are Hill's constants ${ }^{(15)}$, and $L_{0}$ is the length of the static muscle.

Energy per unit time consumed by muscle contraction was determined using Eq. (9).

$$
P=F \cdot v+A \cdot v+A \cdot b
$$


(2) Assuming the same condition applied to isometric contraction $(v=0)$ as in (1), results showed the consumed energy with $v=0$ in Eq. (9), as in Eq. (10). For extensible contraction of $(v<0)$ where a muscle extends, the energy per unit time consumed using a muscle was regarded as the same energy as that consumed for isometric contraction. The consumption energy of a muscle was ascertained using Eq. (10).

$$
P=A \cdot b
$$
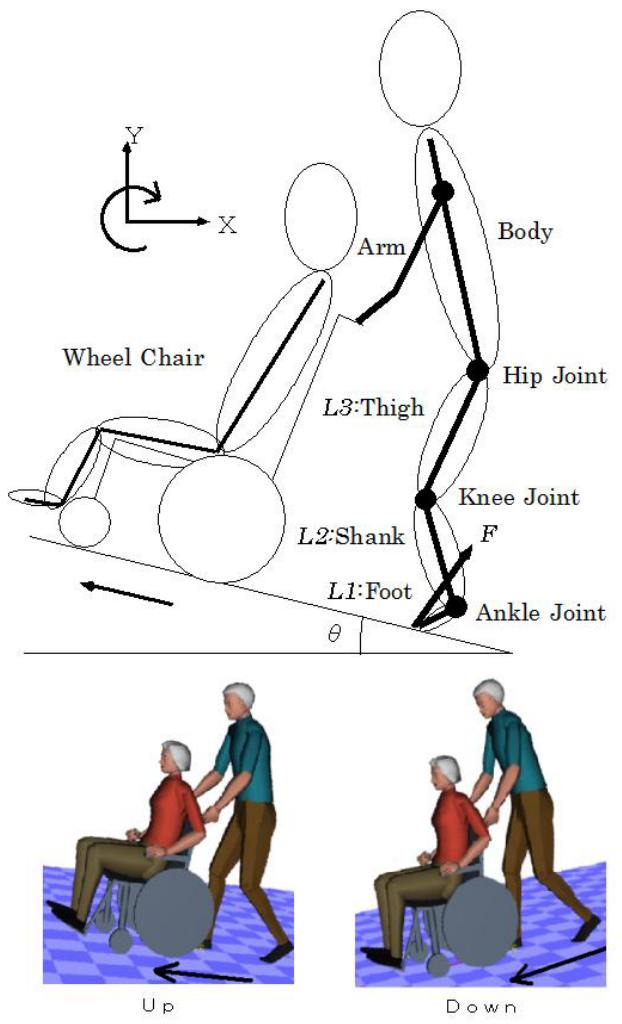

Fig. 1 Link Model

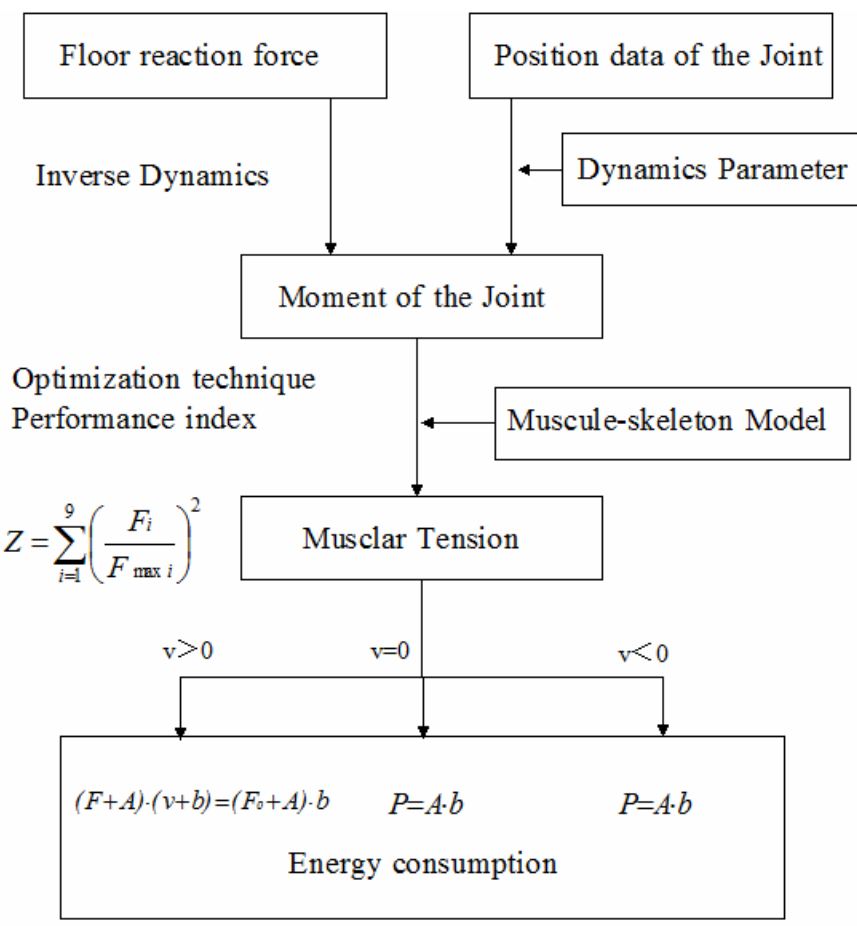

Fig. 3 Block Diagram Showing the Process

(10)

Fig. 2 Muscle-Skeleton Model

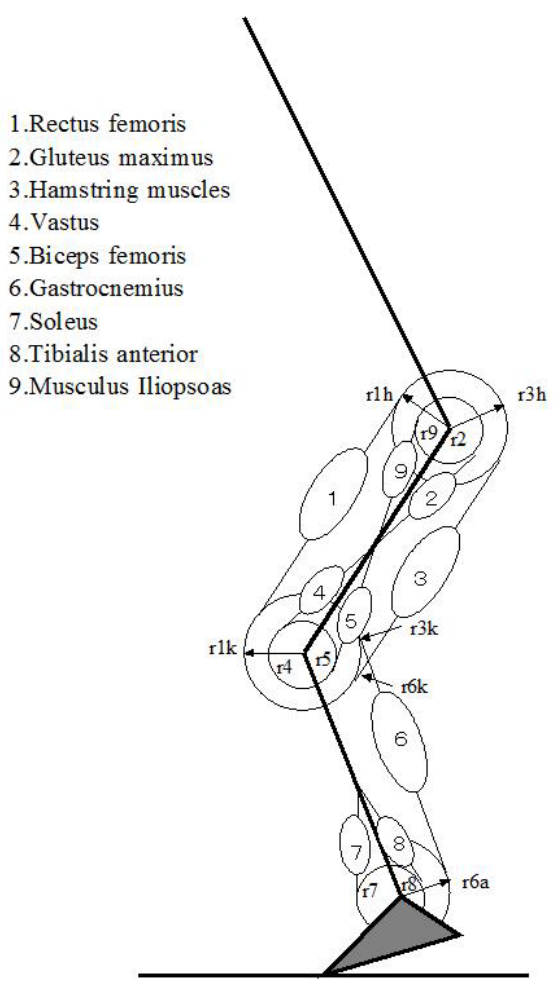


Table 1 Lever Arm Length

\begin{tabular}{|lr|c|}
\hline Lever Arm & & Length $[\mathrm{m}] \times 10^{2}$ \\
\hline Rectus femoris & $r l k$ & 3.5 \\
& $r l h$ & 4.5 \\
Gluteus maximus & $r 2$ & 4.6 \\
Hamstring muscles & $r 3 k$ & 2.8 \\
& $r 3 h$ & 2.9 \\
Vatus & $r 4$ & 3.5 \\
Biceps femoris & $r 5$ & 2.5 \\
Gastrocenemius & $r 6 a$ & 5.1 \\
& $r 6 k$ & 3.6 \\
Soleus & $r 7$ & 5.1 \\
Tibialis anterior & $r 8$ & 5.1 \\
Muscles Iliopsoas & $r 9$ & 4.5 \\
\hline
\end{tabular}

\section{Walking Experiments}

\subsection{Motion Capture}

Motion capture (Vicon140; Oxford Metrics Ltd.) was combined with measurements obtained using space coordinates at the reference point with a force plate (9286; Kistler Instruments $A G)$, which measures the force applied to the floor for motion capture of a subject. The system's measurement precision was $\pm 1.5 \mathrm{~mm}$ at space coordinates, with floor reaction force measurement precision of $\pm 3 \mathrm{~N}$. Four infrared cameras were installed on a 5 $\mathrm{m} \times 5 \mathrm{~m}$ floor, measuring three-dimensional images and floor reaction force data at a sampling frequency of $60 \mathrm{~Hz}$ (Fig. 4). The gait course width was $1.5 \mathrm{~m}$ and the distance of the gait course was $5 \mathrm{~m}$. The slope is $1 / 12$ (8.3\%) (Fig. 5).

\subsection{Experiments}

Subjects were 14 healthy people aged 17-67 years old. A person was fixed in a wheelchair (male, aged 38, $166 \mathrm{~cm}, 60 \mathrm{~kg}$ ). The purpose and contents of experiments were explained to subjects. After informed consent was obtained from them, the experiment was conducted. Two experiments were conducted for slope ascent and descent. Considering the average individual gait, the walking velocity was set to $1.2 \mathrm{~m} / \mathrm{s}$, walking cycle to $1 \mathrm{~s}$, and the step length to $0.63 \mathrm{~m}$. Reference markers were attached to five sites (the fifth metatarsal, ankle joint, knee joint, hip joint and shoulder joint) to measure the walking trajectory.

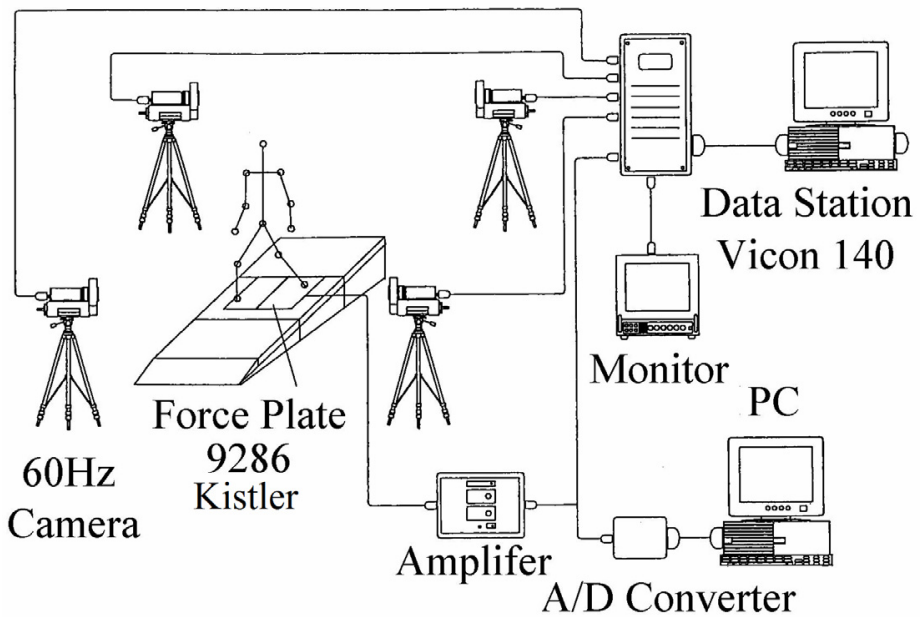

Fig. 4 Experimental Setup 


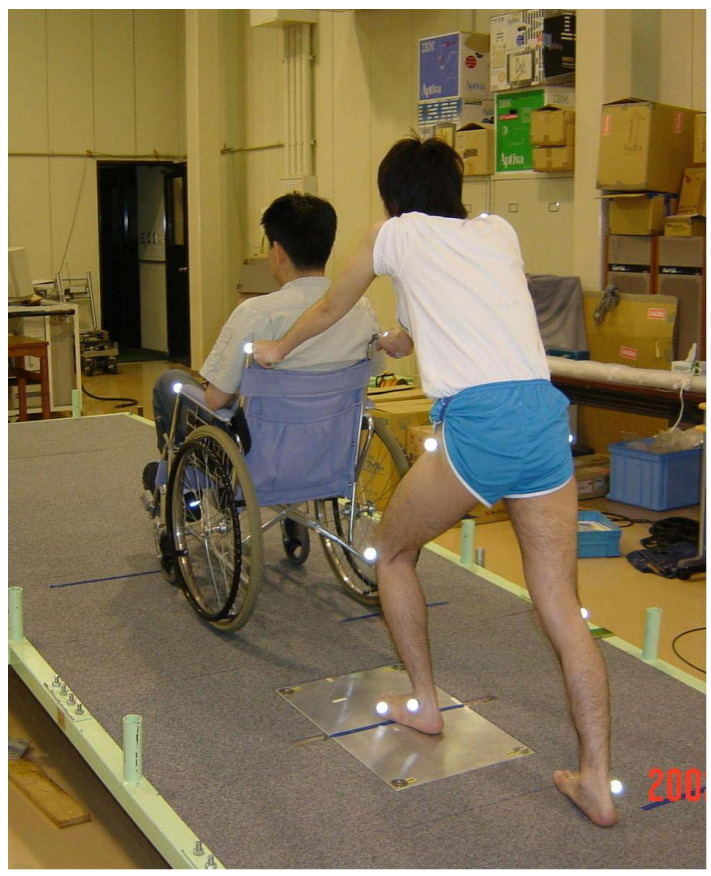

Fig. 5 Gait with Wheelchair

\section{Results and Considerations}

The trajectory and angle of the ankle, knee, and hip and floor reaction force as well as the body's center of gravity, joint moment, and muscle energy consumption were elucidated. Muscle energy consumption was calculated as an index of evaluation of the reduction in the burden on the lower limbs. Results for subject A, a 64-year-old man, $160 \mathrm{~cm}$ tall and weighing $54 \mathrm{~kg}$, are described below (Figs. 6-9). Most subjects produced similar graphs to those shown for ' $A$ '.

\subsection{Trajectory and Moment of Joints}

Figures 6 and 7 show the subject at left walking normally (a) and walking with a wheelchair (b) in slope ascent and descent. The ordinate shows the height from the floor; the abscissa shows the horizontal distance. The movement of the center of gravity is also shown in the figure. A cross $(\times)$ marks the center of gravity. The main points of observation were the trajectory of the shoulder joint and the center of gravity of the body. Periodic fluctuations were observed in the trajectories of the shoulder and center of gravity of the body for both the gaits (Figs. 6, 7). As depicted in Fig. 6, although the helper's back is straight when walking uphill normally on a slope, when walking while assisting the wheelchair, the helper must walk with the waist bent in a forward-leaning posture because the helper must push the wheelchair. In contrast, Fig. 7 portrays a stick diagram of descent on a slope. Both normal walking and walking while assisting the wheelchair show a similar walking form: the helper's back is straight. That occurs because, different from ascending a slope, the wheelchair proceeds by its own weight when descending, necessitating almost no extra effort to push the wheelchair.

Figure 8 shows the moment acting on each joint during a cycle of one step of (a) normal gait and (b) gait with wheelchair in slope ascent. Each moment generated by each joint was made positive for a clockwise rotation. The horizontal axis of Fig. is one-step cycle. From Fig. 8, the joint moments of the ankle and the knee were made just as they were 
for the gait with the wheelchair, and the hip joint moment was made smaller than that of the gait with the wheelchair.

According to results of earlier studies, the burden on the body was evaluated using the moment of each joint. Inoue et al. described in an earlier report ${ }^{(19)}$ that, with the hip joint moment being an index of the burden on muscles at the lumbar, the burden on muscles at the lumbar vertebrae can be evaluated by the peak moment. Yamazaki et al. reported ${ }^{(20)}$ that evaluations of the hip joint moment can serve as a practical evaluation standard for the biomechanical burden on muscles at the lumbar vertebrae.

From Fig. 8, it is apparent that the moments of ankle joint and knee joint were the same in the case of the normal gait and the gait with wheelchair. The hip joint moment increased in value on gait with wheelchair. When walking up the slope, the hip joint moment greatly affected the stance phase, probably because the upper body elderly people tilts forward when walking to ascend a slope.

Figure 9 portrays the moment acting on the ankle, the knee and the hip joint during a cycle of one step of (a) normal gait and (b) gait with wheelchair on a down slope. Each joint moment shows no change.

\subsection{Muscle Energy Consumption}

The muscular energy consumption on the up slope is presented in Fig. 10 for the cases of (a) normal gait and (b) gait with wheelchair, and the muscular energy consumption on the down slope is presented in Fig. 11 for the cases of (a) normal gait and (b) gait with wheelchair. The muscular energy consumption is summed up over nine muscles in the musculoskeletal model presented in Fig. 2. The muscular energy consumption shows the average gait data of 14 subjects. The ordinates of both figures are normalized by the body weight of subjects. The symbol '*' in Fig. 10 corresponds to a statistical level in Student's $t$-test, the symbol ' $* *$ ' corresponds to a $1 \%$ statistical level. The statistical significance test was used for examining the difference of the experimentally obtained results for the normal gait and the gait with wheelchair. On the ascending slope, the muscular energy consumption increased by $13 \%$ for the case of the gait with the wheelchair compared with the normal gait. It was apparently used to push the wheelchair upward. Results show that the significance level for the muscular energy consumption on the up slope was within $1 \%$.

When the experiment was conducted on the down slope, the resultant data of the muscular energy consumption showed no difference between the normal gait and the gait with wheelchair. No statistically significant difference was found. This experiment used a steady gait after five paces.

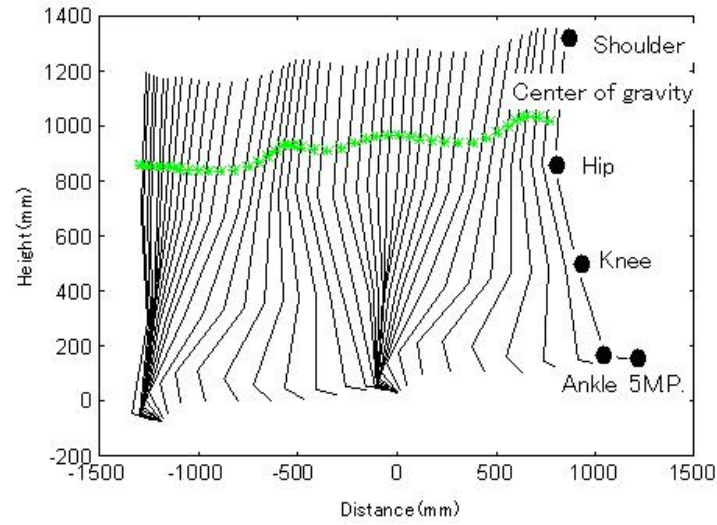

(a) Normal Gait

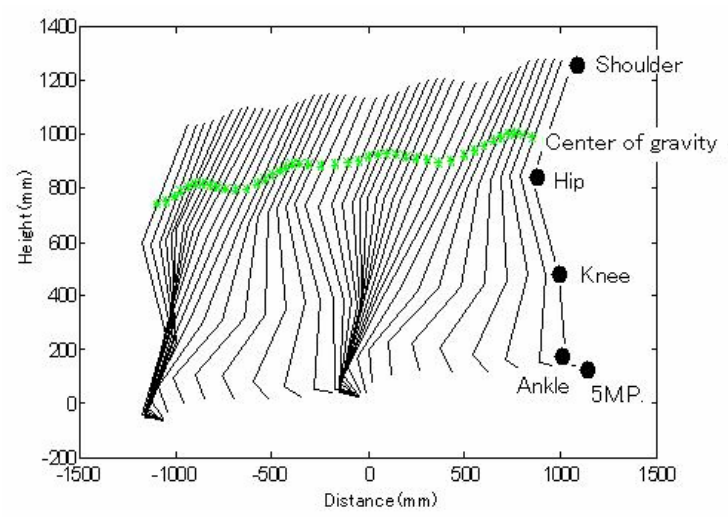

(b) Gait with Wheelchair

Fig. 6 Stick Figure (Up Slope) 


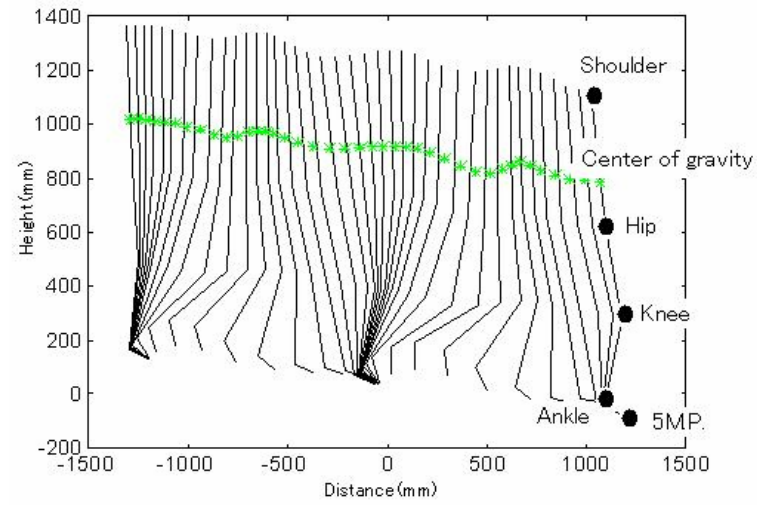

(a) Normal gait

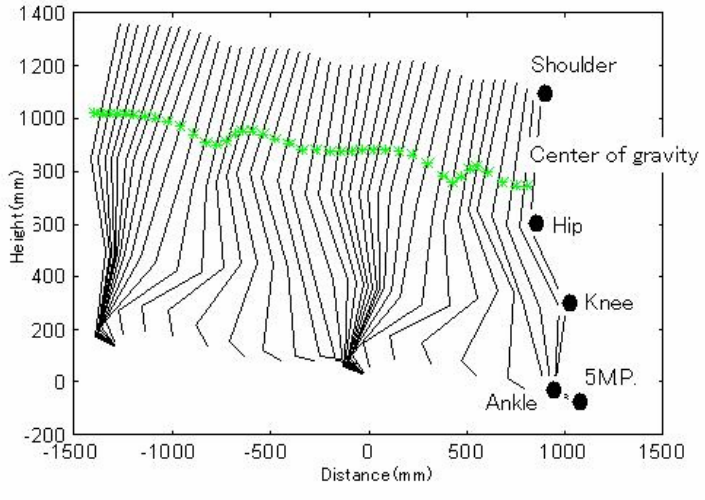

(b) Gait with wheelchair

Fig. 7 Stick Figure (Down Slope)

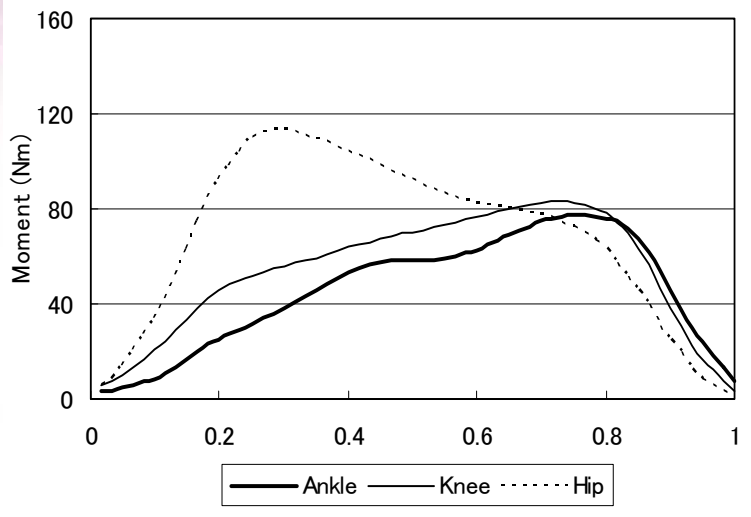

(a) Normal gait

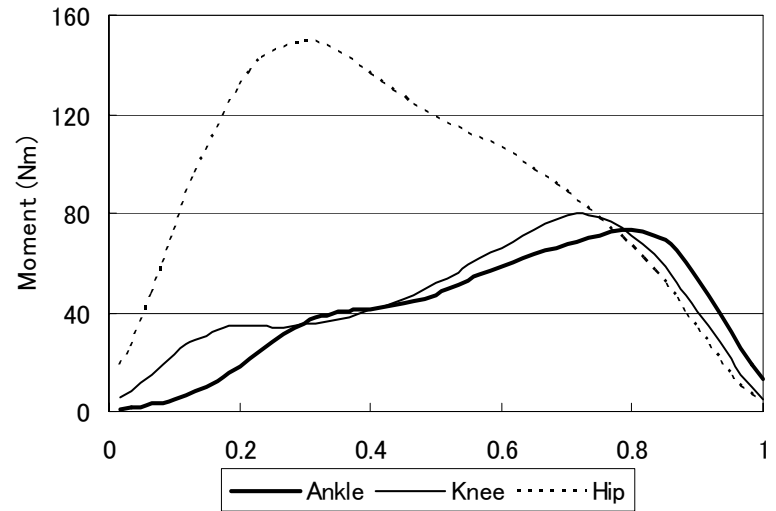

(b) Gait with wheelchair

Fig. 8 Joint Moment (Up Slope)

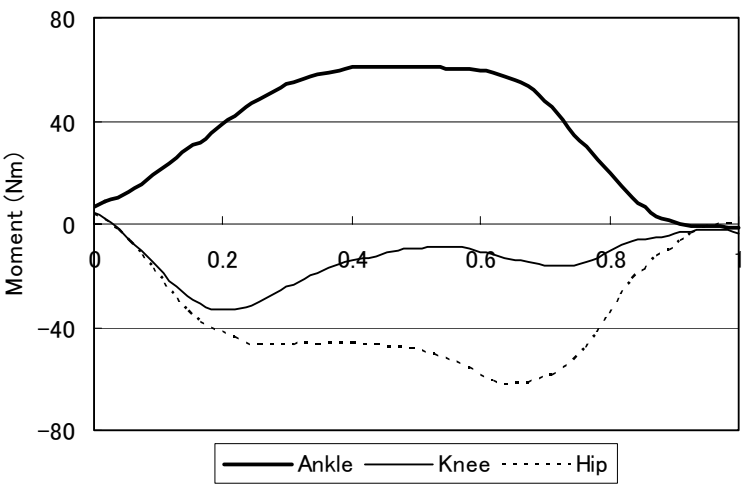

(a) Normal gait

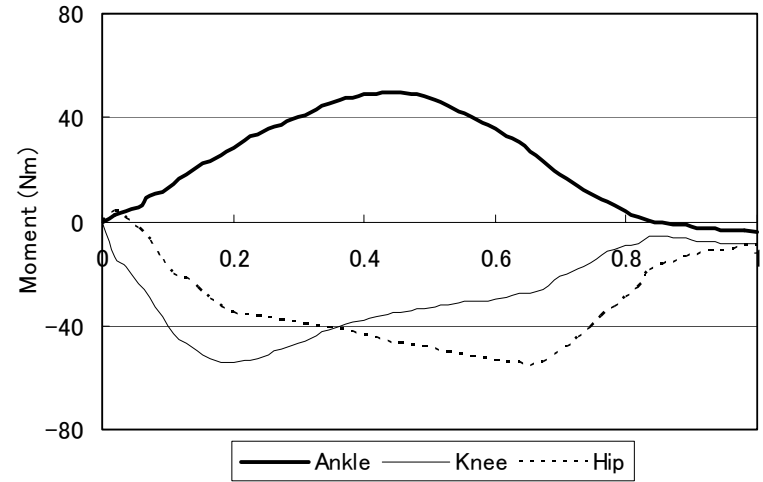

(b) Gait with wheelchair

Fig. 9 Joint Moment (Down Slope) 


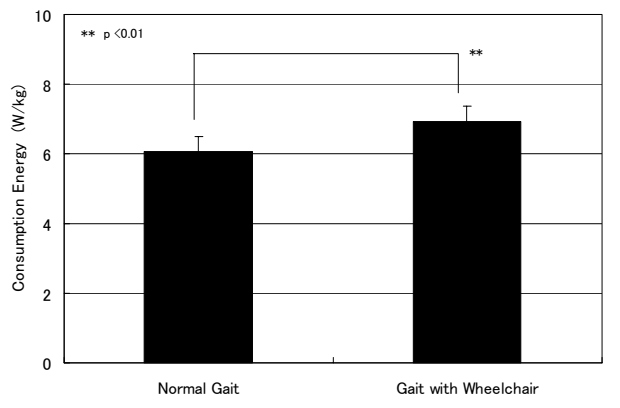

Fig. 10 Energy Consumption (Up Slope)

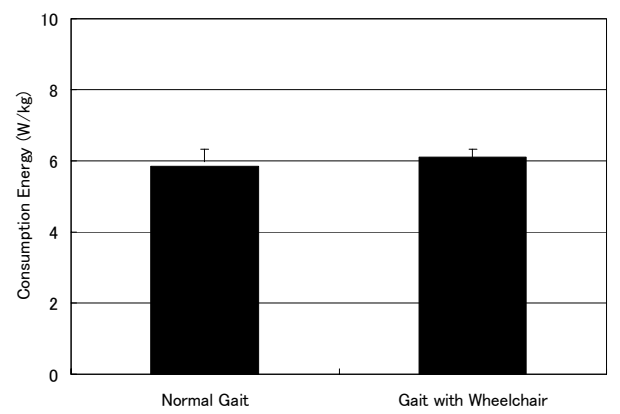

Fig. 11 Energy Consumption (Down Slope)

\subsection{Investigation of evaluation method adequacy}

Using Yamazaki's musculoskeletal model, which is used generally for clinical gait analysis, the ankle joint, knee joint, and hip joint moment were calculated from empirically obtained trajectories of joints and floor reaction force. For estimation of the muscle tension, equations representing balance between muscle tension and moment are three, which are far fewer than the number of muscle forces (nine). Therefore, muscle tension was not determined uniquely. Then, Eq. (7) was used as an evaluation function for the muscle tension. The adequacy of the evaluation function was proven in an earlier study ${ }^{(15)}$ by comparison between the assumed muscle tension and the muscle potential.

Next, the adequacy of muscular consumption energy is described using the model calculation method proposed by Ehara et al. For cases in which the muscle contracts at the muscular contractile rate, muscle causes isometric contraction, and the muscle relaxes. The energy consumption of the lower limb is inferred to be lower with slower walking speed and higher with faster walking speed. In earlier studies ${ }^{(10),(11),(17)}$, Ehara et al. verified its adequacy by comparison between energy consumption obtained from the musculoskeletal model during level walking and energy expended during walking, as obtained empirically from oxygen consumption through exhaled gas analysis. Results of muscular consumption energy evaluation obtained in this study were added to the comparison between model calculation in an earlier study ${ }^{(17)}$ and consumption energy of oxygen consumption obtained empirically. They are presented in Fig. 12. In the figure, $\square$ represents the consumption energy by model calculation by Ehara et al., + represents the consumption energy assumed from oxygen consumption, and - represents the consumption energy evaluated using the method described herein. From Fig. 12, it is considered that because consumption energy assumed by the current study exhibits nearly the same tendency with consumption energy levels of earlier studies, the evaluation method introduced in this study is adequate.

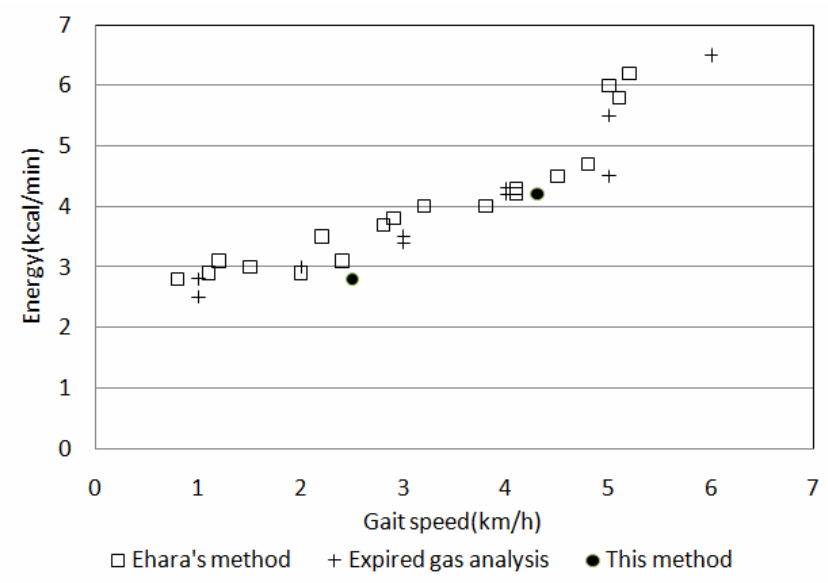

Fig. 12 Comparison of Energy (Flat Gait). 


\section{Conclusions}

This study, which conducted a mechanical assessment of a helper's walking using a wheelchair on a slope, used the Musculoskeletal Model to estimate the joint moment and the consumption energy. The salient results obtained in this study are described below.

1. Comparison of the gait with wheelchair with the normal gait revealed that, although the helper's back is straightened when walking uphill normally on a slope, when walking during assistance with the wheelchair, the helper must walk with the waist bent in a forward-leaning posture because the helper must push the wheelchair. Both normal walking and walking while assisting the wheelchair show a similar walking form: the helper's back is straight. That occurs because, different from ascending a slope, the wheelchair proceeds by its own weight when descending, necessitating almost no extra effort to push the wheelchair.

2. From the experiment results obtained for each joint locus and the floor reaction force, it is apparent that the ankle joint and knee joint moments were the same in the case of the normal gait and the gait with wheelchair. The hip joint moment increased in value for the gait with the wheelchair. In walking up the slope, the hip joint moment strongly affected the stance phase, probably because the helper is walking upward, increasing the load on the hip joint.

3. We estimated lower muscular energy consumption using the musculoskeletal model. In the up slope, the muscular energy consumption increased by $13 \%$ for the case of gait with the wheelchair compared with the normal gait. It was apparently used to push the wheelchair upward. When the experiment was conducted with motion down the slope, the resulting data of the muscular energy consumption showed no difference between the normal gait and that with the wheelchair. This result evaluated the typical nursing care load. By evaluating the burden placed on the body of a helper quantitatively, the care burden was clarified. Consequently, approaches to mitigate or eliminate care processes that impart a great burden to the body can be promoted. These approaches are expected to engender improvement in the quality of care.

This study evaluated the hip joint moment. Future studies will use a rigid link model of the spine, which incorporates the muscles, the discs, and the ligaments to estimate the precise hip joint load. After applying this measuring system, the gait used for pushing a hand truck in a factory will be assessed.

Our great appreciation is extended to the staff of the Akita Research and Technology Center and students of Mechanical Engineering at Akita University.

\section{References}

(1) A. Kakimoto, S. Suzuki and Y. Sekiguchi: Development of Wheelchair for Propelling Ability Evaluation, Life Support Vol. 11, No. 4, (1999), pp.114-119. (in Japanese)

(2) M. Sasaki, T. Iwami, G. Obinata, H. Doki, K. Miyawaki and M. Kinjo: Biomechanics Analysis of the Upper Limb During Wheelchair Propulsion, Transactions of the Japan Society of Mechanical Engineer, Series C Vol. 71, No. 702, (2005), pp. 654-666. (in Japanese)

(3) M. Sasaki, T. Iwami, G. Obinata, K. Miyawaki, H. Miura, Y. Shimada and K. Kiguchi: Analysis of Wheelchair Propulsion and Hand Force Pattern Based on Manipulation Ability of the Upper Limb, Transaction of the Japan Society of Mechanical Engineer, 
Series C Vol. 73, No. 732, (2007), pp. 2279-2286. (in Japanese)

(4) M. Nishioka, I. Yoneda, and A. Okada: A functional reach on maneuverability for attendant propelled wheelchairs Rehabilitation Engineering Vol. 17, No. 2, (2002), pp. 27-30. (in Japanese)

(5) A. Kawaguchi, T. Yukawa, S. Yamamoto and E. Fukui: An evaluation of push handle height of wheelchair, The17th Japanese Conference on the Advancement of Assistive and Rehabilitation Technology (2002), pp. 473-476. (in Japanese)

(6) S. Ko, S. Imanishi, Y. Matsuura, S. Tsubouchi, S. Kayahara, N. Shimizu and M. Matsuura: Psycho-physiological Studies for Attendance of the Persons with Wheelchairs, St. Andrew's University Bulletin of Research Institute, (2002), pp. 49-64.

(7) L.H.V. van der Woude, C.M. van Koningsbruggen, A.L. Krose and I. Kinga: Effect of push handle height on net moment and forces on the musculoskeletal system during standardized wheelchair pushing tasks, Prosthetics and Orthotics International Vol. 19, (1995), pp. 188-201.

(8) H. Uchiyama, T. Suzuki, J. Kurata and Y. Murakami: Investigate and estimate Attendant's Propelling Work Against Wheelchair's Load, Transaction of the Japan Society of Mechanical Engineer, Series C Vol. 72, No. 715, (2006), 842-849. (in Japanese)

(9) N. Yamazaki: Analysis Model and Simulation of the Biped-locomotion, Journal of the Society of Biomechanisms Japan Vol. 3, (1975), 261-269. (in Japanese)

(10) Y. Ehara, M. Beppu and S. Nomura: Muscular efficiency during walking, Journal of the Society of Biomechanisms Japan Vol. 9, (1989), pp.93-104. (in Japanese)

(11) Y. Ehara, M. Beppu, S. Nomura and Y. Kunimi: Estimation of Energy Consumption During Level Walking, Journal of the Society of Biomechanisms Japan Vol. 10, (1990), pp.163-172. (in Japanese)

(12) M. Ohashi and Y. Ehara: Application of Mathematical Model Methods for Gait-analysis, Rehabilitation Vol. 26, No. 11, (1998), pp.1049-1054. (in Japanese)

(13) K. Miyawaki, T. Iwami, G. Obinata, Y. Kondo, K. Kutsuzawa, Y. Ogasawara and S. Nishimura: JSME International Journal Series C Vol. 43, No. 4, (2000), 966-974.

(14) M. Ae, H.P. Tang and T. Yokoi: Estimation of inertia properties of the body segments in Japanese athletes, Journal of the Society of Biomechanisms Japan Vol. 11, (1992), pp.23-33 (in Japanese)

(15) A. Pedotti, V.V. Krishnan and L. Stark: Optimization of Muscle-Force Sequencing in Human Locomotion, Mathematical Biosciences Vol. 38, (1993), pp. 57-76.

(16) H.P. Kunzi, H.G. Tzschach and C.A. Zehnder: Numerische Methoden Der Mathematischen Optimierung mit Algol-und FORTRAN-Programmen, Charles E. Tuttle Co. Inc. Tokyo, (1969), pp. 77-83.

(17) A.V. Hill and R.C. SEC.: The heat of shortening and the dynamic constants of muscle, Proc. Roy. Soc. B (1938), pp.136-195.

(18) Y. Ehara: Dynamic model of muscle and estimate of muscle tension. Japanese Society of Biomechanics Text (1994), pp.33-57. (in Japanese)

(19) T. Inoue, G. Fernie and P.L. Santaguida: Lower Back Loads During Maneuvering Tasks with Lifting Devices, Journal of the Society of Biomechanisms Japan Vol. 15, (2000), pp.243-254. (in Japanese)

(20) N. Yamazaki, S. Yamamoto and T. Inoue: Measurement of Transferring Motions and Evaluation of Caregiver's Lower-Back Load, Journal of the Society of Biomechanisms Japan Vol. 16, (2002), pp.195-205. (in Japanese) 